\title{
Brief Report: Human peritoneal mesothelial cells display phagocytic and antigen- presenting functions to contribute to intraperitoneal immunity
}

Tanya J Shaw, $\mathrm{PhD}^{\mathrm{a} * \wedge}$, Xiang Y Zhang, $\mathrm{MD}^{\mathrm{b} *}, \mathrm{ZM}$ Huo $\mathrm{MD}^{\mathrm{c}}$, David Robertson ${ }^{\mathrm{d}}$, Patricia A Lovell ${ }^{\mathrm{e}}$, Angus G Dalgleish, $\mathrm{MD}^{\mathrm{c}}$, Desmond PJ Barton, $\mathrm{MD}^{\mathrm{b}}$

\section{AFFILIATIONS}

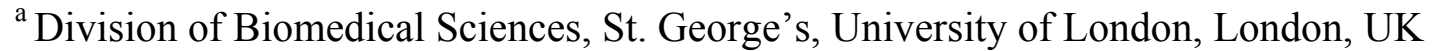

${ }^{b}$ Department of Obstetrics and Gynaecology, St. George's Healthcare NHS Trust

${ }^{\mathrm{c}}$ Division of Infection and Immunity, St. George's, University of London, London, UK

${ }^{d}$ Department of Pathology, Breakthrough Breast Cancer Centre, Institute of Cancer Research, London, UK

${ }^{\mathrm{e}}$ Royal Marsden Hospital, London, UK

* These authors contributed equally.

${ }^{\wedge}$ Now at King's College London - Centre for Molecular and Cellular Biology of Inflammation, London, UK

Corresponding author: Dr Tanya Shaw

PRE-PUBLICATION King's College London

Centre for Molecular and Cellular Biology of Inflammation

1st Floor, New Hunt's House, Room 1.32D

London SE1 1UL

United Kingdom

tanya.shaw@kcl.ac.uk

Tel: (+44) 02078486475

Corresponding author: $\quad$ Mr Desmond PJ Barton

POST-PUBLICATION Division of Gynaecological Oncology

(including for reprints) Dept. of Obstetrics and Gynaecology

St. George's Hospital

Blackshaw Road

London SW17 0QT

dbarton@sgul.ac.uk

Tel: (+44) 02087250188

Sources of support: St George's, University of London 


\title{
BRIEF REPORT
}

\begin{abstract}
Mesothelial cells lining the peritoneal cavity are strategically positioned to respond to and counter intraperitoneal infections, cancer cells, and other challenges. We have investigated human peritoneal mesothelial cells (HPMCs) for phagocytic activity, expression of surface Major Histocompatibility Complex (MHC) Class II and accessory molecules involved in antigen presentation, and the ability to present recall antigens to $\mathrm{T}$ cells. Phagocytosis of dextran, latex beads and Escherichia coli was observed by flow cytometry, and internalization was visualised using confocal and electron microscopy. Flow cytometry and/or cellular ELISA showed constitutive expression of ICAM-I, LFA-3, and B7-1, but not B7-2 or MHC II. Interferon-gamma induced MHC II and ICAM-1 expression in a dose- and time-dependent manner. Importantly, HPMCs induced autologous $\mathrm{CD}^{+} \mathrm{T}$ lymphocyte proliferation $\left({ }^{3} \mathrm{H}\right.$-incorporation) after pulse with recall antigen. HPMCs equipped with phagocytic and antigen-presenting machinery are anticipated to have an integral role in intraperitoneal immune surveillance.
\end{abstract}




\section{BRIEF REPORT}

\section{INTRODUCTION}

The peritoneal cavity encounters and responds to damaging cell types, microorganisms, and foreign antigens, which include ovarian cancer metastases, intraperitonal infection, peritoneal dialysis, and endometriosis. It is lined with an epithelial monolayer, the mesothelium, formed by human peritoneal mesothelial cells (HPMCs) and contains resident and migratory immunological cells - macrophages, T cells, and natural killer (NK) cells. ${ }^{1}$ Historically, it was thought innate immune cells provided the first line of defense, but evidence suggests HPMCs may have a significant immunological role. $^{2}$

Under certain conditions, non-immune cell types such as fibroblasts ${ }^{3}$ and endothelial cells ${ }^{4}$ display immune functions including release of pro-inflammatory cytokines and chemokines, phagocytosis of particles and microorganisms, and antigen presentation. Some recent reports indicate that HPMCs have antigen presenting capacity, phagocytic activity, 5,6 and can express Major Histocompatibility Complex (MHC) class II and intracellular adhesion molecule (ICAM). ${ }^{7,8}$

Herein we report that HPMCs show the classical properties of accessory cells for Tcell responses - phagocytic activity, induction of MHC II, and expression of accessory molecules ICAM-1, LFA-3, and B7-1. Functionally, HPMCs were capable of recall antigeninduced autologous $\mathrm{T}$ cell activation, represented by $\mathrm{T}$ lymphocyte proliferation.

\section{MATERIALS AND METHODS}

All reagents are from Sigma Aldrich (Poole, UK) unless stated otherwise.

Primary HPMCs (passage 2-4), derived from normal human omentum biopsies (study approved by local Research Ethics Committee) were maintained as described. ${ }^{9,}{ }^{10}$ The following cells were used for comparison purposes: HMrSV5, immortalised human peritoneal mesothelium cells (gift from R Piedagnel ${ }^{9}$ ); mouse ovarian surface epithelial (mOSE) cells 


\section{BRIEF REPORT}

(gift from BC Vanderhyden ${ }^{10}$ ), SKOV3 ovarian cancer cell line (European Collection of Cell Cultures, ECACC), THP-1 human monocytic leukaemia cell line (ECACC) pre-treated for 24h with phorbol 12-myristate 13-acetate (PMA, 100ng/ml), and human umbilical vein endothelial cells (HUVECs, ECACC). Peripheral blood mononuclear cells (PBMCs) from healthy donors were isolated by Histopaque density gradient centrifugation. T lymphocytes were enriched magnetically (Pan T Cell Isolation Kit, Miltenyi Biotec, Surrey, UK).

To measure phagocytic uptake activity, HPMCs in suspension were incubated in complete media (CM) containing: 1) FITC-dextran; 2) polystyrene red fluorescent latex beads $(\varnothing, 0.8 \mu \mathrm{m})$; or 3) E. coli. Uptake was quantified using flow cytometry [FACScan, Becton Dickinson; data analysis: Cellquest ${ }^{\mathrm{TM}}$ (BDIS, San Jose, CA) and/or FlowJo software (Tree Star, Inc.)]. Expression of cell surface antigens was also detected by flow cytometry (Table 1).

To visualise phagocytic uptake, HPMCs were cultured on coverslips to $70-80 \%$ confluence, then exposed to latex beads or green fluorescent E.coli for 8h. For confocal microscopy (Leica TCS/SP2), cells were fixed (4\% paraformaldehyde, $1 \mathrm{~h})$, permeabilised with $0.5 \%$ Triton X-100, and counterstained with AlexaFluor546-phalloidin (F-actin), and To-PRO-3 (nuclei, Molecular Probes). For electron microscopy (EM, Phillips CM100BIO), cells were fixed in 2.5\% gluteraldehyde in 0.1 M Sörensen's Phosphate buffer $\left(\mathrm{pH} 7.4 ; 4^{\circ} \mathrm{C}\right.$ overnight), post-fixed ( $2 \mathrm{~h}$, room temperature) in $1 \%$ osmium tetroxide, dehydrated, and resinembedded. Transverse sections of monolayers were stained with uranyl acetate and lead citrate prior to visualisation.

For Cellular Enzyme-Linked Immunosorbent Assay (C-ELISA), HPMCs cultured with various stimuli were fixed $(0.5 \%$ periodate-lysine-parafomaldehyde), permeabilised (0.1\% saponin), and then incubated with primary antibodies (1h). The Vectastain Universal Elite ABC kit (Vector Laboratories) was used for visualization (OD490nm). Subsequent 


\section{BRIEF REPORT}

staining with $0.08 \%$ crystal violet, and measurement of OD550nm determined total cells/well. Western blotting was conducted according to standard protocol (Merck Millipore), using precast polyacrylamide $8-16 \%$ gradient tris-glycine gels (Generon, UK) that were transferred to nitrocellulose membrane. Blots were blocked in 5\% milk and blotted for ICAM-1 (Santa Cruz Biotechnology, Inc., USA) or glyceraldehyde-3-phosphate dehydrogenase (GAPDH; Abcam, UK). Horseradish peroxidase-conjugated secondary antibodies were used, and results were visualised using a Bio-Rad ChemiDoc MP system.

To measure accessory cell-dependent antigen-induced $\mathrm{T}$ cell proliferation, $\mathrm{CD}^{+} \mathrm{T}$ cells in RPMI1640 with 10\% human AB serum (10\% $/$ well) were added to 96-well roundbottomed plates. Autologous HPMCs were irradiated (50Gy), washed, and added to the above plates in a dilution series $\left(1 \times 10^{4}\right.$ to $1.25 \times 10^{3} /$ well). Tuberculin purified protein derivatives (PPD, $500 \mathrm{IU} / \mathrm{ml}$ ) were applied to all test wells (or omitted for the control), and cells cultured for $6 \mathrm{~d}$. Cells were labelled for the final $16 \mathrm{~h}$ with $1.0 \mu \mathrm{Ci} /$ well $\left[{ }^{3} \mathrm{H}\right]$ thymidine (ICN Biomedicals, Inc.), harvested onto glass fibre filter mats, and the incorporated radioactivity measured (Wallac 1450 microbeta liquid scintillation counter).

\section{RESULTS}

Incorporation of FITC-dextran, latex beads, and E. coli by HPMC

To investigate phagocytic capabilities, HPMC were incubated with fluorescentlytagged dextran particles or polystyrene latex beads (Fig. 1A). Flow cytometry revealed strong fluorescent signal in $\sim 80 \%$ of cells incubated with FITC-dextran for $30 \mathrm{~min}$, and in $\sim 50 \%$ of cells incubated with latex beads, whereas there was minimal uptake in cells incubated on ice. Time-course analysis of incorporation of latex beads showed that mean fluorescent intensity (MFI) fluctuated at baseline levels for $2 \mathrm{~h}$, then significantly increased, at which time the percentage of cells having engulfed beads also increased, again peaking near $50 \%$ at the 


\section{BRIEF REPORT}

conclusion of the $24 \mathrm{~h}$ experiment (Fig. 1B). This heterogeneity in phagocytic capabilities was observed to a comparable extent in the HMrSV5 mesothelial cell line, as well as THP-1 and HUVEC cells (Fig. 1C). Mouse ovarian surface epithelial cells and the SKOV3 ovarian cancer cell line however, were much more uniformly capable of engulfment ( $\sim 90 \%$, Fig $1 \mathrm{C})$. Confocal and EM were used to verify that HPMCs had internalised the particles (Fig. 1D, EM; Fig. 1E, confocal microscopy).

To further understand the phagocytic mechanisms of HPMCs, cells were first analysed by flow cytometry for the expression of human immunoglobulin Fc $\gamma$ receptors. None of our HPMC cells expressed detectable levels of CD16 (FcRIII), CD32 (FcRII), or CD64 (FcRI)(data not shown), and when $E$. coli were pre-opsonised with $10 \%$ pooled human serum the rate of phagocytosis was unchanged (data not shown). Also, cells were negative for

mannose receptor (a C-type lectin that also mediates pre-phagocytosis binding; ${ }^{11}$ data not shown), suggesting alternative machinery is responsible for the uptake.

To investigate the ability of HPMCs to provide antigen specific signals for the activation of naïve T cells we studied the membrane expression of MHC molecules by FC and C-ELISA. High levels of cell surface MHC I (HLA-A,B,C) were demonstrated in resting HPMCs, whereas MHC II (HLA-DP,DR,DQ) expression was not detected in the basal state (Fig. 2A). However, HLA-DP,DR,DQ was induced by pre-treatment with $100 \mathrm{U} / \mathrm{ml}$ of IFN- $\gamma$ for $72 \mathrm{~h}$, but not by TNF- $\alpha$, IL-1 $\beta$ or LPS (Fig. $2 \mathrm{~B}$ ). A time-course experiment showed MHC II induction as early as $24 \mathrm{~h}$, and persistence of expression beyond $96 \mathrm{~h}$, even after withdrawal of IFN- $\gamma$ (data not shown).

The ability of HPMCs to activate $\mathrm{T}$ cells was explored first by examining their constitutive expression of co-stimulatory molecules by flow cytometry. High levels of ICAM1, and intermediate levels of LFA-3 and B7-1 were expressed, but the expression of B7-2 was negligible (Fig. 2C). Modulation of their expression by cytokines (IFN- $\gamma$, TNF $\alpha$, IL1 $\beta$, or 


\section{BRIEF REPORT}

LPS) was assessed by C-ELISA, showing that only ICAM-1 expression (not LFA-3 or B71/2) was modulated by treatments, and only IFN- $\gamma$ could induce its expression. The IFN- $\gamma-$ inducible increase in ICAM-1 expression was significant at $4 \mathrm{~h}$, near maximum at $24 \mathrm{~h}$, persisted for at least 72h (Fig. 2D) and was concentration-dependent (Fig. 2E). Western blotting indicated that ICAM-1 induction by IFN- $\gamma$ occurred to a comparable extent in other similar cell lineages, HUVECs and SKOV3, when considered alongside HMrSV5 (Fig. 2F).

The potential of normal HPMCs to present antigen was evaluated with an autologous T cell proliferation assay. HPMCs were co-cultured with autologous CD3 $+\mathrm{T}$ lymphocytes purified from the peripheral blood of PPD immunized individuals. These cells induced HPMC proliferation $\left(\left[{ }^{3} \mathrm{H}\right]\right.$ thymidine-incorporation) in response to recall antigen (PPD) in a cell ratiodependent manner, which at the highest HPMC:T cell ratio of 1:10 was significantly enhanced relative to the control without PPD (Fig. 2G).

\section{DISCUSSION}

Intraperitoneal cancer, surgery, and infection can breach the sterile homeostatic environment of the peritoneal cavity; accordingly, there are a number of defense mechanisms. In addition to the immune cells in the peritoneal fluid, the peritoneal monolayer (HPMCs) also provide a line of defense: it is a semi-permeable physical barrier, with anti-inflammatory activity. ${ }^{12}$ Intuitively, HPMCs should have an immunological role, and we and others have shown that they can produce many immunomodulatory cytokines and growth factors. ${ }^{13}$ Our data now add to other evidence that HPMCs are phagocytic and have many of the features of a non-professional antigen-presenting cell (APC).

The phagocytic activity of HPMCs substantiate other reports that HPMCs can engulf microorganisms and apoptotic cancer cells.$^{5,6,14}$ That only $\sim 50 \%$ of cells engulfed particles, a level seemingly on par with other cell lineages including monocyte-derived cells and 


\section{BRIEF REPORT}

endothelial cells, interestingly suggests HPMCs may be a functionally heterogeneous population, with a range of maturation or activation states.

Phagocytosis is a receptor-mediated process initiated when a foreign particle binds to an opsonin receptor such as Fc $\gamma$ or CR3. ${ }^{11}$ Alternatively, there are opsonin-independent mechanisms including C-type lectin mediated binding. ${ }^{11}$ As reported by others, ${ }^{6,14}$ Fcy receptor expression was undetectable on HMPC cultures, and correspondingly, opsonisation did not enhance particle uptake. Mannose receptor expression was also undetectable; however, other non-opsonin receptors (e.g. Stab2, Ax1, Tyro3) were identified to be expressed by HPMCs by Wagner et al. ${ }^{6}$ These alternative strategies used by nonprofessional phagocytes are effective, but likely less efficient with a narrower target particle range. $^{15}$

The quantitative level of surface MHC expression is a determinant of antigenpresenting capacity; as expected, we found resting HPMCs constitutively expressed MHC I molecules, but there was no detectable MHC II. However, this could be induced by IFN- $\gamma$ in a time- and concentration-dependent manner, as reported by Hausmann et al. ${ }^{7}$ In contrast, Valle et al reported that some primary cultures do express resting levels, ${ }^{8}$ which may reflect the health status of the donor.

A second signal critical for T-cell activation is typically derived from co-stimulatory molecules [e.g. ICAM-1 (CD54), LFA-3 (CD58), and B7-1 (CD80)]. ${ }^{16}$ We show that HPMCs constitutively expressed intermediate levels of surface ICAM-1 and LFA-3, and low levels of B7-1. Moreover, ICAM-1 expression was up-regulated by IFN- $\gamma$ stimulation in a time- and concentration-dependent manner, with minimal influence by TNF- $\alpha$ or IL-1 $\beta$. This IFN- $\gamma$ induced enhancement of ICAM-1 has similarly been observed by others in mesothelium, ${ }^{7,8}$ and was shown here to be a feature shared by endothelial and SKOV3 ovarian cancer cells. Mesothelial LFA-3 and B7-1 expression has not been previously recognised however. 


\section{BRIEF REPORT}

With the essential molecular prerequisites on HPMCs, we next demonstrated their functionality: HPMCs efficiently processed and presented recall antigen to autologous T cells. Interestingly, this occurred in the absence of additional IFN- $\gamma$, and was not augmented by further IFN- $\gamma$. One possible explanation is that unstimulated HPMCs expressed low levels of MHC II, undetected here but sufficient to induce antigen-specific proliferative $\mathrm{T}$ cell responses. Alternatively, the HPMCs and/or T-cells may have produced IFN- $\gamma$ or an alternative signal in the co-culture, which in turn induced maximal MHC II and ICAM-1 expression.

In conclusion, normal HPMCs have a significant inherent phagocytic activity, can be induced to produce MHC II, and constitutively express co-stimulatory molecules. Moreover, they can present recall antigen to autologous memory $\mathrm{T}$ lymphocytes. Characterization of the role(s) of these amateur phagocytes in local host defense mechanisms will improve our understanding of the consequences of damage to this tissue lining and may lead to new therapies for intraperitoneal diseases.

\section{References}

[1] Broche F, Tellado JM. Defense mechanisms of the peritoneal cavity. Curr Opin Crit Care. 2001;7: 105-16.

[2] Topley N. The host's initial response to peritoneal infection: the pivotal role of the mesothelial cell. Perit Dial Int. 1995;15: 116-7.

[3] Arlein WJ, Shearer JD, Caldwell MD. Continuity between wound macrophage and fibroblast phenotype: analysis of wound fibroblast phagocytosis. Am J Physiol. 1998;275: R1041-8.

[4] Dini L, Lentini A, Diez GD et al. Phagocytosis of apoptotic bodies by liver endothelial cells. J Cell Sci. 1995;108 ( Pt 3): 967-73. 


\section{BRIEF REPORT}

[5] Visser CE, Brouwer-Steenbergen JJ, Schadee-Eestermans IL et al. Ingestion of Staphylococcus aureus, Staphylococcus epidermidis, and Escherichia coli by human peritoneal mesothelial cells. Infect Immun. 1996;64: 3425-8.

[6] Wagner BJ, Lindau D, Ripper D et al. Phagocytosis of dying tumor cells by human peritoneal mesothelial cells. J Cell Sci. 2011;124: 1644-54.

[7] Hausmann MJ, Rogachev B, Weiler M et al. Accessory role of human peritoneal mesothelial cells in antigen presentation and T-cell growth. Kidney Int. 2000;57: 476-86. [8] Valle MT, Degl'Innocenti ML, Bertelli R et al. Antigen-presenting function of human peritoneum mesothelial cells. Clin Exp Immunol. 1995;101: 172-6.

[9] Rougier JP, Moullier P, Piedagnel R, Ronco PM. Hyperosmolality suppresses but TGF beta 1 increases MMP9 in human peritoneal mesothelial cells. Kidney Int. 1997;51: 337-47. [10] Clark-Knowles KV, Garson K, Jonkers J, Vanderhyden BC. Conditional inactivation of Brca1 in the mouse ovarian surface epithelium results in an increase in preneoplastic changes. Experimental cell research. 2007;313: 133-45.

[11] Groves E, Dart AE, Covarelli V, Caron E. Molecular mechanisms of phagocytic uptake in mammalian cells. Cell Mol Life Sci. 2008;65: 1957-76.

[12] Mutsaers SE, Whitaker D, Papadimitriou JM. Stimulation of mesothelial cell proliferation by exudate macrophages enhances serosal wound healing in a murine model. $\mathrm{Am}$ J Pathol. 2002;160: 681-92.

[13] Zhang XY, Guckian M, Nasiri N et al. Normal and SV40 transfected human peritoneal mesothelial cells produce IL-6 and IL-8: implication for gynaecological disease. Clin Exp Immunol. 2002;129: 288-96.

[14] Muijsken MA, Heezius HJ, Verhoef J, Verbrugh HA. Role of mesothelial cells in peritoneal antibacterial defence. J Clin Pathol. 1991;44: 600-4. 


\section{BRIEF REPORT}

[15] Bronson R. Is the oocyte a non-professional phagocyte? Hum Reprod Update. 1998;4: $763-75$

[16] Glik A, Douvdevani A. T lymphocytes: the "cellular" arm of acquired immunity in the peritoneum. Peritoneal dialysis international : journal of the International Society for Peritoneal Dialysis. 2006;26: 438-48.

\section{Figure legends}

Fig. 1 Human peritoneal mesothelial cells (HPMCs) phagocytose dextran, latex beads and E. coli. (A) Primary HPMCs, in suspension, were incubated on ice $\left(4^{\circ} \mathrm{C}\right.$, blue) or at $37^{\circ} \mathrm{C}$ (red) with FITC-dextran (Sigma, MW 43,200 Da, 1mg/ml) for 30min, or red fluorescent polystyrene latex beads (ø, $0.88 \mu \mathrm{m}, 10: 1$ particle:cell) for $24 \mathrm{~h}$. The uptake was detected by flow cytometry (5000 cells analysed). (B) Percentage of cells containing beads (left y axis, black) and mean fluorescent intensity (MFI) per cell (representing internalised beads, right y axis, blue) are plotted against incubation time ( $\mathrm{n} \geq 3$, representative experiment shown). (C) For comparison, HMrSV5, THP-1, HUVEC, mOSE and SKOV3 were also incubated in suspension with FITC-dextran on ice (blue) or at $37^{\circ} \mathrm{C}$ (red) for $2 \mathrm{~h}$, and analysed by flow cytometry. (D) HPMCs incubated with latex beads for $8 \mathrm{~h}$ at $37^{\circ} \mathrm{C}$ were processed for electron microscopy (scale bar 5um). (E) HPMCs incubated with E. coli (K-12 strain, AlexaFluor488conjugated, Molecular Probes; 10:1 particle:cell) for 8h were fixed in 4\% paraformaldehyde, and stained with AlexaFluor546 phalloidin (red) and To-PRO-3 (nuclear stain, blue). Medial transverse (G, top) and medial sagittal sections (G, bottom) are shown (scale bar $10 \mu \mathrm{m})$.

Fig. 2. HPMCs express MHC Class I and II molecules, co-stimulatory molecules and have antigen-presenting capacity. (A, C) Representative flow cytometric results of resting HPMCs. (A) Surface membrane HLA-A,B,C were detected, but not HLA-DP,DR,DQ. (C) Surface membrane ICAM-1 (CD54), LFA3 (CD58), and B7-1 (CD80) were detected, but not 


\section{BRIEF REPORT}

B7-2 (CD86). Red lines show isotype controls, black lines represent specific antibody staining ( $\mathrm{n} \geq 3$, representative experiment shown). (B, D, E) Representative C-ELISA results of (B) HLA-DP,DR,DQ or (D, E) ICAM-1 expression in response to: IFN- $\gamma(100 \mathrm{U} / \mathrm{ml})$ or TNF- $\alpha(100 \mathrm{U} / \mathrm{ml})$ or IL-1 $\beta(10 \mathrm{ng} / \mathrm{ml})$ or LPS $(1 \mu \mathrm{g} / \mathrm{ml}$; E. coli serotype 0127:B8; Sigma), or (E) IFN- $\gamma(0-1000 \mathrm{U} / \mathrm{ml})$ for $72 \mathrm{~h}$. Graphs represents mean $\pm \mathrm{SD}$ of duplicate measurements from three experiments with two HPMC lines. Data are expressed as ratios of OD490 to OD550 to normalise for cells/well. (F) SKOV3, HMrSV5 and HUVEC cells cultured \pm IFN- $\gamma$ (100 U/ml) for $24 \mathrm{~h}$ were analysed by western blot for ICAM-1 and GAPDH (loading control). (G) Irradiated (50Gy) HPMCs $\left(1 \times 10^{4}\right.$ to $1.25 \times 10^{3} /$ well in 96 -well plates) were co-incubated with autologous CD3+ T lymphocytes $\left(10^{5} /\right.$ well) at a HPMC:T cell ratio ranging from 1:80 to $1: 10 \pm$ recall antigen (tuberculin purified protein derivate, PPD, $500 \mathrm{IU} / \mathrm{ml}$ ). Control ratio (no PPD) was $1: 10$. Proliferation was measured by $\left[{ }^{3} \mathrm{H}\right]$ thymidine uptake $(1.0 \mu \mathrm{Ci} /$ well $)$ over final $16 \mathrm{~h}$ of a $6 \mathrm{~d}$ co-culture; data are presented as the average counts per minute $(\mathrm{cpm})$ of the triplicate wells of a representative experiment (conducted in duplicate with cells from four donors). Significance was assessed by ANOVA with Tukey's multiple comparison test (significance inferred at $\mathrm{p}<0.05, *$, GraphPad Prism). 
Figure 1

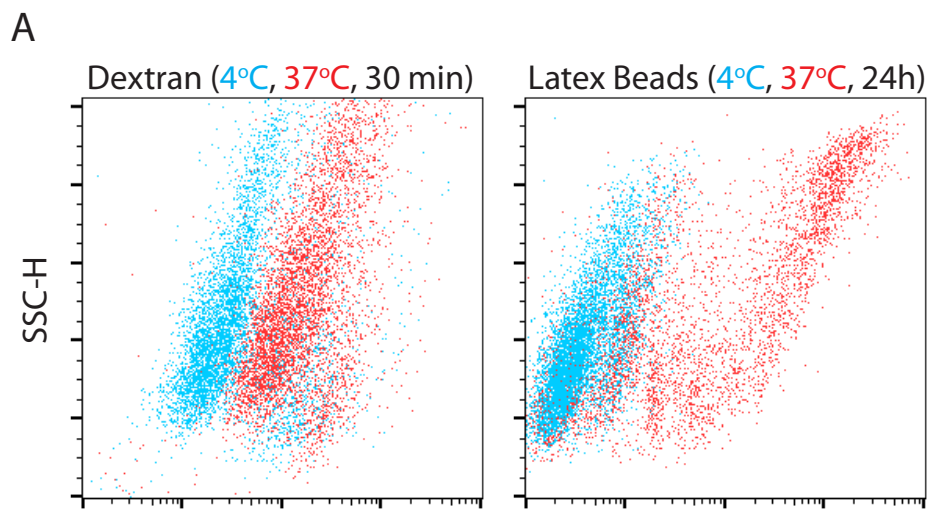

FITC-H

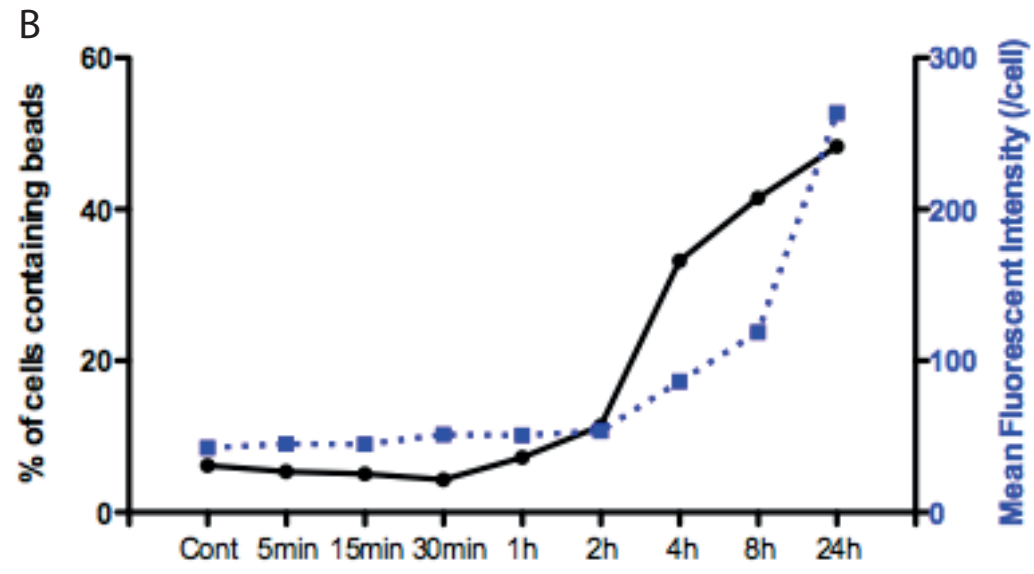

C - Dextran $\left(4^{\circ} \mathrm{C}, 37^{\circ} \mathrm{C}, 2 \mathrm{~h}\right)$
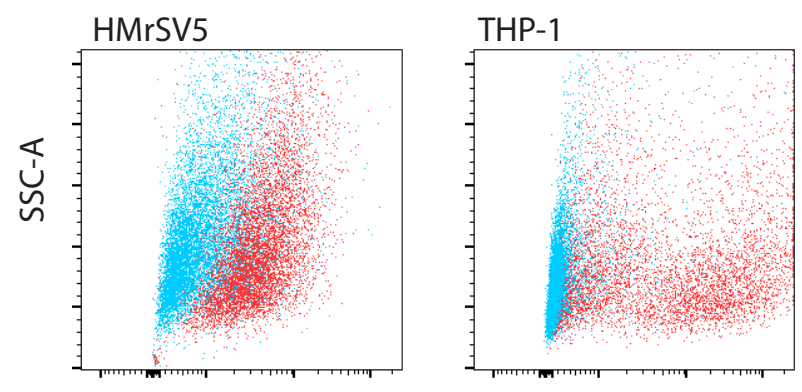

HUVEC
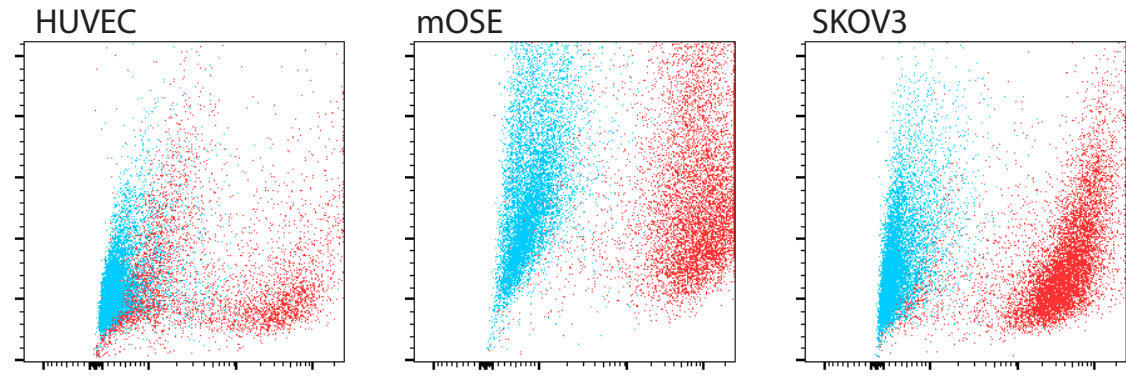

FITC-A

$\mathrm{D}-37^{\circ} \mathrm{C}$ (Beads, 8h)

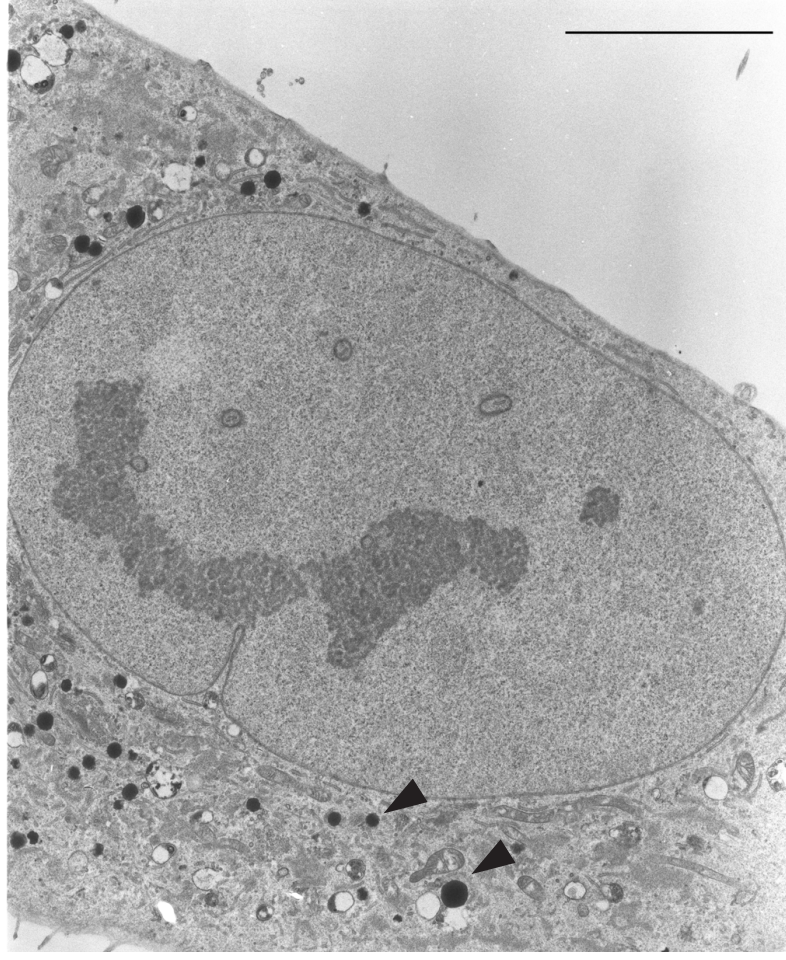

$\mathrm{E}-37^{\circ} \mathrm{C}$ (E. coli, 8h)

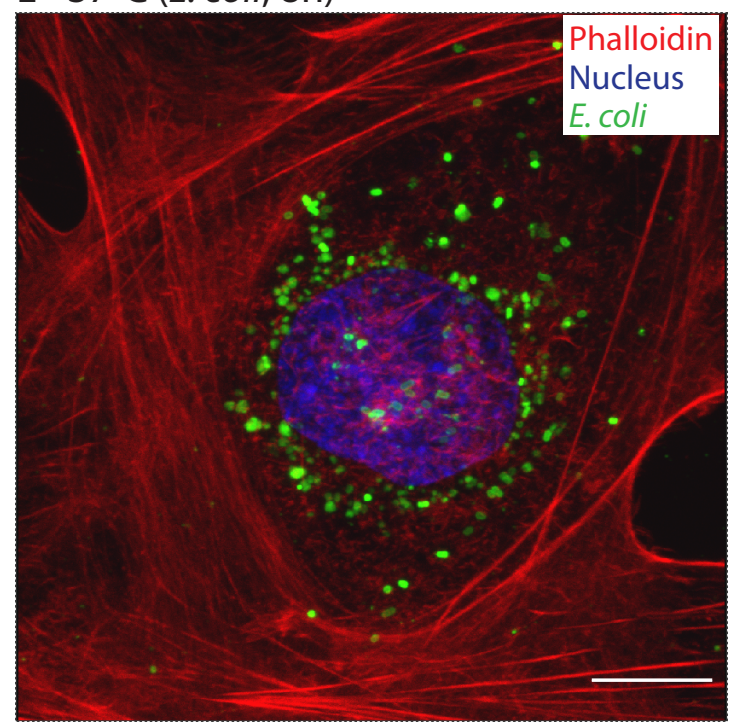

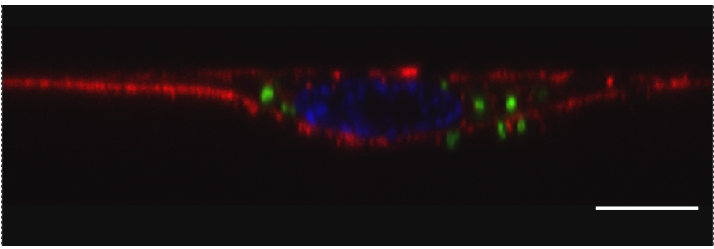


Figure 2

A

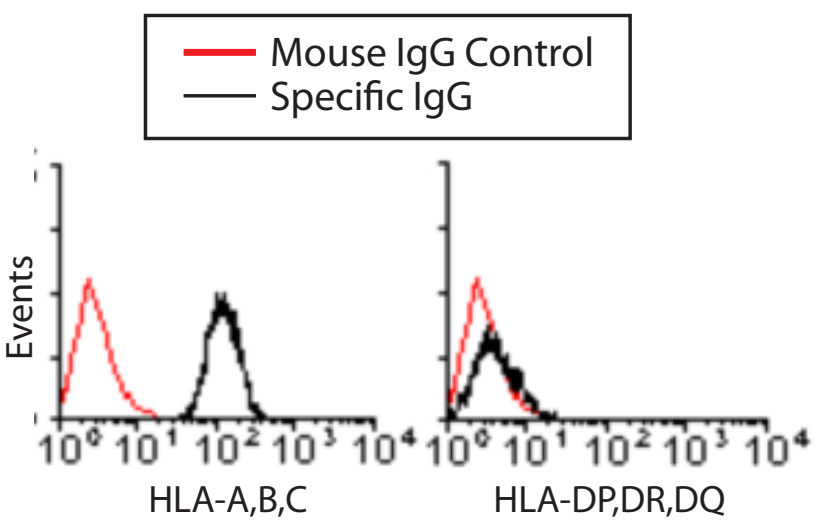

C

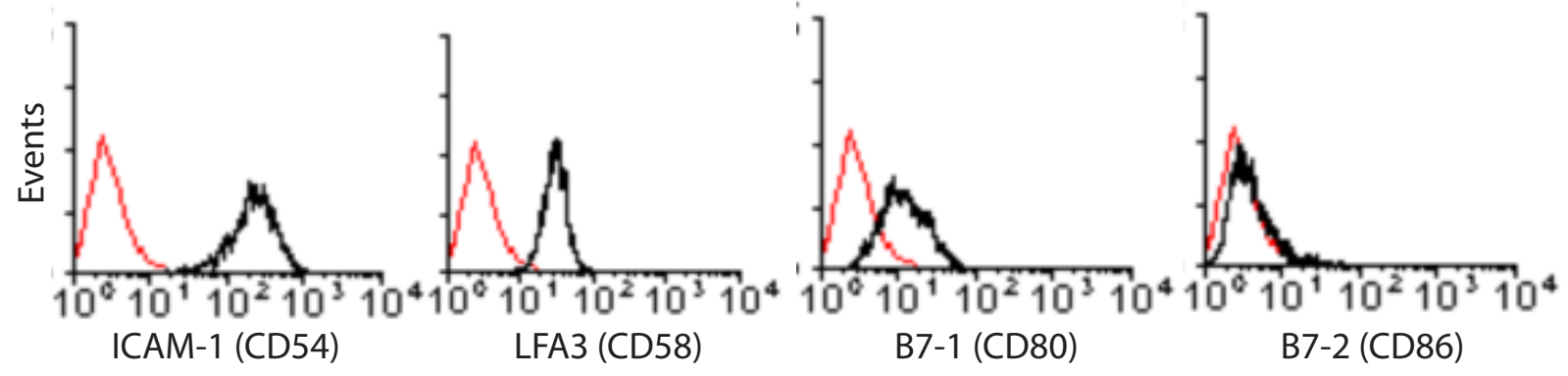

D

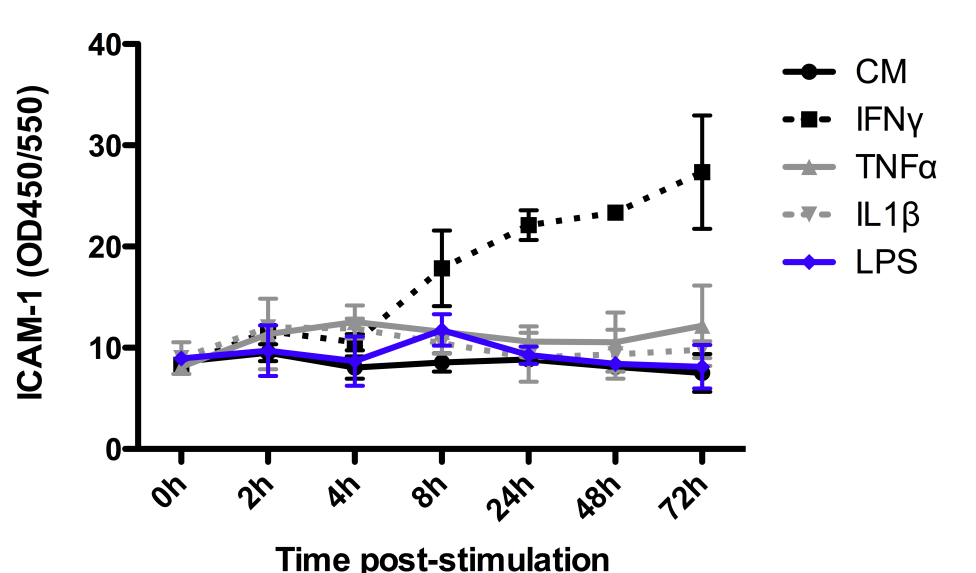

F

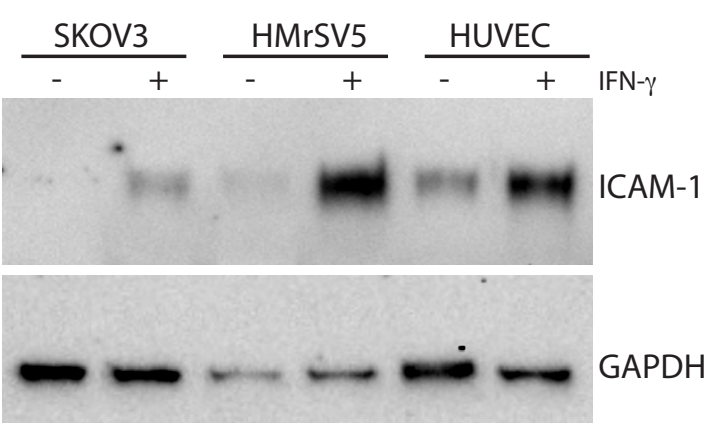

B

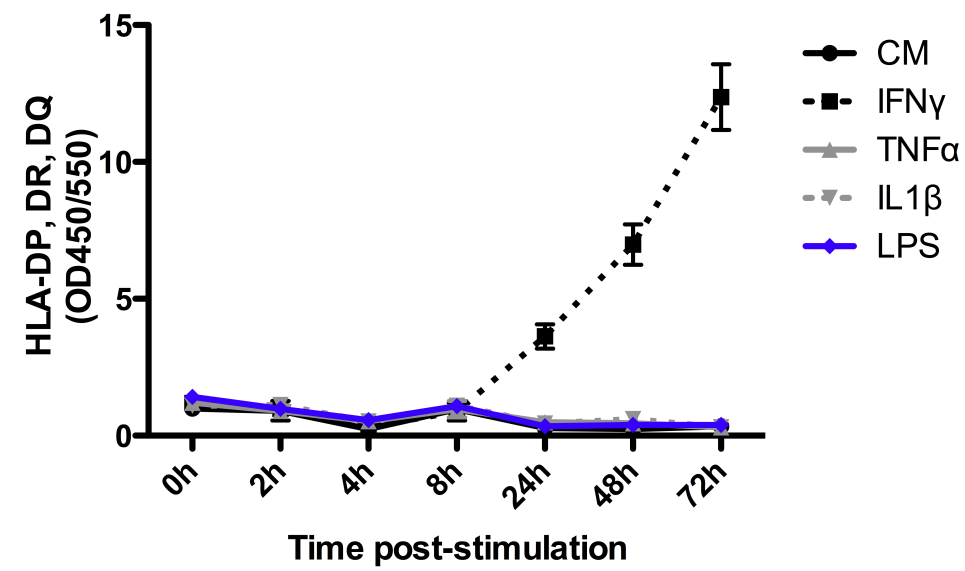

E

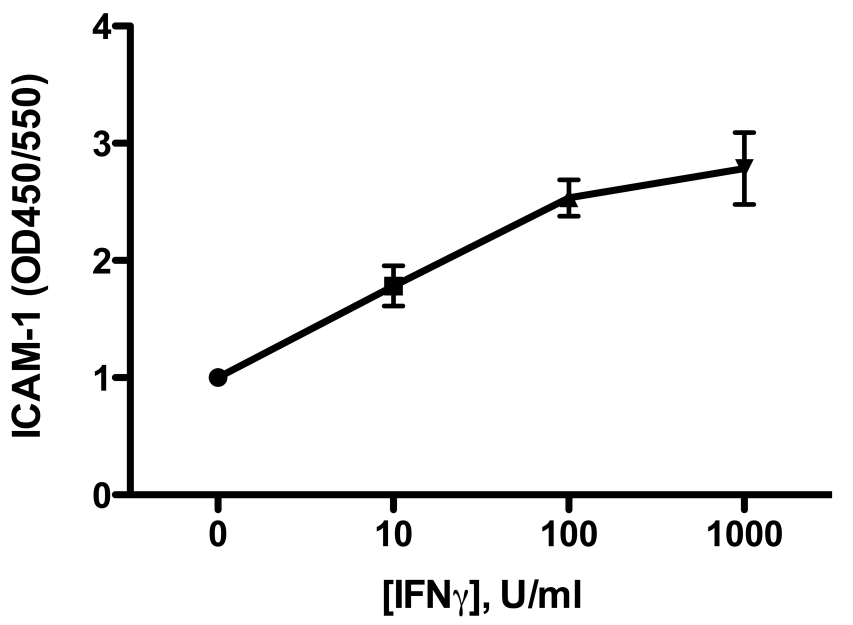

G

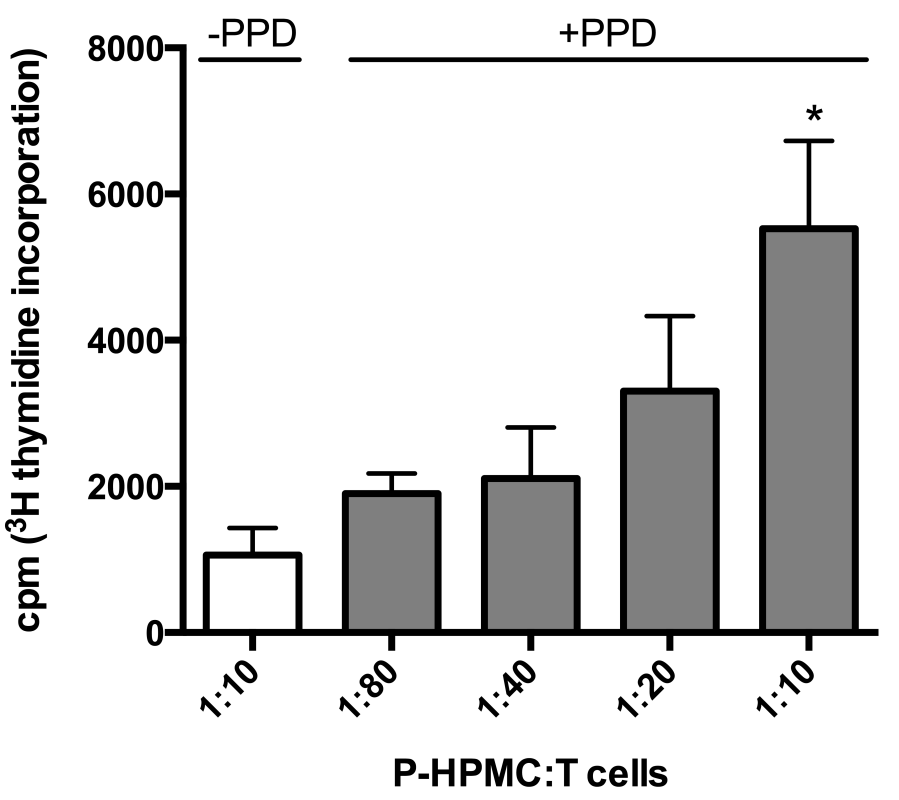


Table 1. Antibodies used for analyses.

\begin{tabular}{|lll|}
\hline Antigen & Unconjugated, Supplier & Conjugate, Supplier \\
\hline ICAM-1 (CD54) & BD Bioscience & FITC, Serotec \\
LFA-3 (CD58) & BD Bioscience & FITC, Serotec \\
HLA-ABC & BD Bioscience & FITC, Serotec \\
HLA-DP,DQ,DR & BD Bioscience & FITC, Serotec \\
CD64 (Clone 10.1) & $\mathrm{n} / \mathrm{a}$ & FITC, BD Bioscience \\
CD206 (mannose & $\mathrm{n} / \mathrm{a}$ & FITC, BD Bioscience \\
receptor)(Clone 19.2) & & \\
B7-1 (CD80) & BD Bioscience & PE, Serotec \\
B7-2 (CD86) & n/a & PE, Serotec \\
CD14 (Clone TUK4) & n/a & PE, DAKO \\
CD16 (Clone DJ130c) & n/a & PE, DAKO \\
CD3 & n/a & PerCP, BD Bioscience \\
CD32 (Clone 3D3) & BD Bioscience & n/a \\
\hline
\end{tabular}

